\title{
NILPOTENTS IN FINITE SYMMETRIC INVERSE SEMIGROUPS
}

\author{
by GRACINDA M. S. GOMES* and JOHN M. HOWIE
}

(Received 17th October 1985)

\section{Introduction}

In semigroup theory as in other algebraic theories a significant part of the total effort is appropriately applied to the study of certain standard examples occurring, as it were, "in nature". The most obvious such semigroup is the full transformation semigroup $\mathscr{T}(X)$ (see [3]) and about this semigroup a great deal is known in both the finite and infinite cases.

Inverse semigroups (see [3, Chapter V]) are of interest not only as a naturally occurring special case of semigroups but also for their role in describing partial symmetries. Mathematically this property is expressed by the Vagner-Preston Theorem [3, Theorem V.1.10], by which every (finite) inverse semigroup is embedded in an appropriate (finite) symmetric inverse semigroup $\mathscr{I}(X)$, consisting of all partial one-to-one maps, or subpermutations (to use an attractive term from Cameron and Deza [1]) of $X$.

Since the theory of inverse semigroups is now extensive enough to have been the subject of a substantial book by Petrich [7], it is perhaps rather surprising that very little has been written on the symmetric inverse semigroup. Certainly the Green equivalences are well understood. (See [3, Example V.2].) A notable contribution was made by Liber [5], whose description of the congruences on $\mathscr{I}(X)$ is analogous to Mal'cev's work [6, see also 2, Section 10.8] on full transformation semigroups. Much more recently Howie and Marques-Smith [4] investigated certain properties of nilpotent elements in $\mathscr{I}(X)$ in the case where $X$ is infinite. This paper is to some extent motivated by that work.

Let $Z=(1, \ldots, n)$, let $I_{n}(=\mathscr{I}(Z))$ be the symmetric inverse semigroup on $Z$, let $S_{n}$ be the symmetric group on $Z$ and let $S P_{n}=I_{n} \backslash S_{n}$, the inverse semigroup of all proper subpermutations of $Z$.

The inverse semigroup $S P_{n}$ has $n \mathscr{J}$-classes (or $\mathscr{D}$-classes, since $\mathscr{J}=\mathscr{D}$ ) $J_{0}, \ldots, J_{n-1}$, where $J_{r}(r=0, \ldots, n-1)$ consists of all subpermutations of height $r$, i.e. all $\alpha$ in $S P_{n}$ for which

$$
|\operatorname{dom} \alpha|=|\operatorname{im} \alpha|=r .
$$

Notice that $J_{0}$ consists solely of 0 , the unique subpermutation with empty domain, and that

$$
\left|J_{r}\right|=\left(\begin{array}{l}
n \\
r
\end{array}\right)^{2} r !
$$

*Research supported by Instituto Nacional de Investigação Cientifica, Portugal. 
In particular $\left|J_{n-1}\right|=n^{2}(n-1)$ ! The $\mathscr{D}$-class $J_{n-1}$ contains $n \mathscr{R}$-classes $R_{i}$ (corresponding to the $n$ different possible domains $Z \backslash\{i\}$ of cardinality $n-1)$ and $n \mathscr{L}$-classes $L_{j}$ (corresponding to the $n$ different possible images $Z \backslash\{j\}$ ). Each of the $n^{2} \mathscr{H}$-classes $H_{i, j}=R_{i} \cap L_{j}$ contains $(n-1)$ ! elements.

Let $N$ be the set of nilpotent elements in $S P_{n}$ and let $N_{1}=N \cap J_{n-1}$. For a given subset $A$ of $S P_{n}$ we write $\langle A\rangle$ for the inverse subsemigroup of $S P_{n}$ generated by $A$. The main result (Theorem 3.18) of Section 3 is that if $n$ is even then

$$
\langle N\rangle=\left\langle N_{1}\right\rangle=S P_{n}
$$

By contrast, if $n$ is odd we still have $\langle N\rangle=\left\langle N_{1}\right\rangle$, but $\langle N\rangle$ is now a proper inverse subsemigroup $S P_{n} \backslash K$ of $S P_{n}$, where $K$ consists of exactly half of the elements of $J_{n-1}$. Theorem 3.18 also specifies precisely which half of $J_{n-1}$ lies in $\langle N\rangle$.

Since $S P_{n}$ is finite, the ascent

$$
N \subseteq N \cup N^{2} \subseteq N \cup N^{2} \cup N^{3} \subseteq \cdots
$$

must stabilize at some $k$, the least integer for which

$$
N \subseteq N^{2} \cup \cdots \cup N^{k}=\langle N\rangle \text {. }
$$

It is shown in Section 4 that $k=2$ or 3 according as $n$ is odd or even. By contrast, if $l$ is the least integer for which

$$
N_{1} \cup N_{1}^{2} \cup \cdots \cup N_{1}^{l}=\left\langle N_{1}\right\rangle(=\langle N\rangle)
$$

we find that $l=n$.

\section{Nilpotents in a finite symmetric inverse semigroup}

For notation and for basic properties of symmetric inverse semigroups, see $[3$, Section V.1].

An element $\alpha$ of $I_{n}$ is called nilpotent if $\alpha^{k}=0$ for some $k \geqq 1$. The index (of nilpotency) $i(\alpha)$ of $\alpha(\neq 0)$ is the unique $k$ for which $\alpha^{k}=0, \alpha^{k-1} \neq 0$.

It is evident that all nilpotents in $I_{n}$ lie in $S P_{n}$. The first step in our investigation is to give a set-theoretic characterization of nilpotent elements.

Lemma 2.1. Let $\alpha \in J_{r}$, with $r<n$. Then $\alpha$ is nilpotent if and only if there exists no nonempty subset $A$ of $\operatorname{dom} \alpha$ such that $A \alpha=A$.

Proof. If $\alpha=0$ (the empty map) the result is trivial. We may therefore confine ourselves to elements $\alpha$ for which $\operatorname{dom} \alpha \neq \varnothing$. Certainly if there exists $A \neq \varnothing$ inside $\operatorname{dom} \alpha$ for which $A \alpha=A$ we have

$$
A=A \alpha=A \alpha^{2}=\cdots
$$

and so $\alpha$ is not nilpotent. 
Conversely, suppose that no such $A$ exists. Then

$$
\operatorname{im} \alpha=(\operatorname{dom} \alpha) \alpha \neq \operatorname{dom} \alpha
$$

and so $\operatorname{dom} \alpha^{2} \subset \operatorname{dom} \alpha$ (properly). We now show that for $k=2,3, \ldots$

$$
\operatorname{dom} \alpha^{k} \neq \varnothing \Rightarrow \operatorname{dom} \alpha^{k+1} \subset \operatorname{dom} \alpha^{k} .
$$

For suppose by way of contradiction that

$$
\operatorname{dom} \alpha^{k+1}=\operatorname{dom} \alpha^{k} \neq \varnothing
$$

for some $k \geqq 2$. Then

$$
\operatorname{dom} \alpha^{k}=\operatorname{dom}\left(\alpha \cdot \alpha^{k}\right)=\left(\operatorname{im} \alpha \cap \operatorname{dom} \alpha^{k}\right) \alpha^{-1}
$$

Thus $\left|\operatorname{im} \alpha \cap \operatorname{dom} \alpha^{k}\right|=\left|\operatorname{dom} \alpha^{k}\right|$ and so, since the sets are finite,

$$
\operatorname{im} \alpha \cap \operatorname{dom} \alpha^{k}=\operatorname{dom} \alpha^{k}
$$

Fom (2.2) and (2.3) it now follows that

$$
\left(\operatorname{dom} \alpha^{k}\right) \alpha=\operatorname{dom} \alpha^{k}
$$

contrary to hypothesis. We thus have a strict descent

$$
\operatorname{dom} \alpha \supset \operatorname{dom} \alpha^{2} \supset \cdots
$$

and hence there exists $m \geqq 1$ such that $\operatorname{dom} \alpha^{m}=\varnothing$, i.e. such that $\alpha^{m}=0$.

Recall that if $\alpha \in J$, we say that $\alpha$ is of height $r$; let us alternatively write $h(\alpha)=r$. It is clear that for all $\alpha, \beta$ in $I_{n}$

$$
h(\alpha \beta) \leqq \max \{h(\alpha), h(\beta)\}
$$

On the other hand $h(\alpha \beta)$ cannot be too small:

Lemma 2.5. If $\alpha, \beta \in I_{n}$ then

$$
h(\alpha \beta) \geqq h(\alpha)+h(\beta)-n
$$


Proof. Suppose that $\operatorname{dom} \alpha=A, \operatorname{im} \alpha=B, \operatorname{dom} \beta=C, \operatorname{im} \beta=D, \operatorname{dom} \alpha \beta=E=(B \cap C) \alpha^{-1}$, $\operatorname{im} \alpha \beta=F=(B \cap C) \beta$. Then

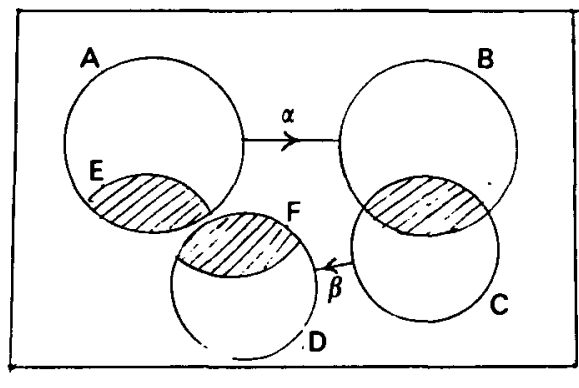

$Z \backslash \operatorname{dom} \alpha \beta=Z \backslash E=(Z \backslash A) \cup(A \backslash E)$.

Thus

$$
\begin{aligned}
\mid Z \backslash \text { dom } \alpha \beta \mid & =|Z \backslash A|+|A \backslash E| \\
& =|Z \backslash A|+|B \backslash(B \cap C)| \text { (since } \alpha \text { is one-one) } \\
& \leqq|Z \backslash A|+|Z \backslash C| .
\end{aligned}
$$

Hence $n-h(\alpha \beta) \leqq n-h(\alpha)+n-h(\beta)$ and the result then follows.

As a consequence,

$$
\alpha \in N_{1}^{k} \Rightarrow h(\alpha) \geqq k(n-1)-n(k-1)=n-k
$$

Lemma 2.5 also has consequences for indices of nilpotency:

Corollary 2.6. If $\alpha$ is a nilpotent of height $r$ in $S P_{n}$ then $i(\alpha) \geqq n /(n-r)$.

Proof. From the lemma it is easy to show inductively that for $k=1,2, \ldots$

$$
h\left(\alpha^{k}\right) \geqq k r-(k-1) n
$$

In particular, if $i(\alpha)=p$ then

$$
0=h\left(\alpha^{p}\right) \geqq p r-(p-1) n
$$

and the result then follows by rearrangement.

Specializing still further we have

Corollary 2.7. If $\alpha$ is a nilpotent of height $n-1$ in $S P_{n}$ then $i(\alpha) \geqq n$. 
Let $r \in\{1, \ldots, n-1\}$ and let $a_{1}, \ldots, a_{r+1}$ be distinct elements of $Z=\{1, \ldots, n\}$. The element

$$
\left(\begin{array}{llll}
a_{1} & a_{2} & \ldots & a_{r} \\
a_{2} & a_{3} & & a_{r+1}
\end{array}\right)
$$

(mapping $a_{i}$ to $a_{i+1}(i=1, \ldots, r)$ ) is clearly nilpotent of index $r+1$. Let us write it as $\left\|a_{1} a_{2} \ldots a_{r+1}\right\|$ and call it a primitive nilpotent in $S P_{n}$.

Theorem 2.8. Every non-zero nilpotent $\alpha$ in $S P_{n}$ is a disjoint union $\alpha_{1} \cup \cdots \cup \alpha_{k}$ of primitive nilpotents. Moreover, $k \leqq n-h(\alpha)$ and

$$
i(\alpha)=\max \left\{i\left(\alpha_{1}\right), \ldots, i\left(\alpha_{k}\right)\right\} .
$$

Proof. Certainly $\operatorname{dom} \alpha \neq(\operatorname{dom} \alpha) \alpha=\operatorname{im} \alpha$ by Lemma 2.1. Let $a_{1} \in \operatorname{dom} \alpha \backslash \operatorname{im} \alpha$ and consider the sequence

$$
a_{1}, a_{2}=a_{1} \alpha, \quad a_{3}=a_{2} \alpha, \ldots
$$

The sequence terminates when we reach an $a_{r+1}=a_{r} \alpha$ such that $a_{r+1} \notin \operatorname{dom} \alpha$. There can be no repetitions in the sequence: if $a_{i}=a_{i+j}=a_{i} \alpha^{j} \quad(j>0)$ then the non-empty set $\left\{a_{i}, \ldots, a_{i+j-1}\right\}$ is invariant under $\alpha$, which is impossible by Lemma 2.1 . Hence the sequence must terminate in the way described.

If $r=h(\alpha)$ then $\alpha$ is the primitive nilpotent $\left\|a_{1} a_{2} \ldots a_{r+1}\right\|$. Otherwise $\alpha$ is a disjoint union

$$
\alpha=\left\|a_{1} a_{2} \ldots a_{r+1}\right\| \cup \beta
$$

Since

$$
\alpha^{m}=\left\|a_{1} a_{2} \ldots a_{r+1}\right\|^{m} \cup \beta^{m}
$$

for $m=1,2, \ldots$ it follows that $\beta$ is nilpotent. But $h(\beta)<h(\alpha)$ and so we may suppose inductively that $\beta$ is a disjoint union of primitive nilpotents. Hence we may express $\alpha$ as $\alpha_{1} \cup \cdots \cup \alpha_{k}$ as required.

Since each $\alpha_{i}$ is of the form $\left\|a_{1} a_{2} \ldots a_{r+1}\right\|$ where $a_{r+1} \notin \operatorname{dom} \alpha$ it follows that there are at least $k$ elements not in $\operatorname{dom} \alpha$; thus

$$
k \leqq n-h(\alpha)
$$

The final assertion of the theorem follows from the fact that

$$
\alpha^{m}=\alpha_{1}^{m} \cup \cdots \cup \alpha_{k}^{m}
$$

for $m=1,2, \ldots$ 
Corollary 2.9. Every nilpotent of height $n-1$ in $S P_{n}$ is primitive and hence of index $n$.

Proof. If $\alpha$ is nilpotent of height $n-1$ then $k \leqq n-(n-1)$ by Theorem 2.8 . Hence $\alpha$ is primitive.

In the introduction the structure of the top $\mathscr{J}$-class $J_{n-1}$ in $S P_{n}$ was described. It can now be seen that each $\mathscr{H}$-class $H_{i, j}(i \neq j)$ contains $(n-1)$ ! elements of which exactly $(n-2)$ ! are (primitive) nilpotents of the form

$$
\left\|j a_{2} \ldots a_{n-1} i\right\|
$$

with $a_{2}, \ldots, a_{n-1} \in Z \backslash\{i, j\}$. Since there are $n(n-1) \mathscr{H}$-classes containing nilpotents, the total number of nilpotents in $J_{n-1}$ is $n$ !.

We end this section with a result on conjugates of nilpotents

Theorem 2.10. If $\beta$ is a nilpotent in $S P_{n}$ and $\alpha \in S P_{n}$ then $\alpha^{-1} \beta \alpha$ is a nilpotent.

Proof. Suppose that $\beta^{k}=0$. Then by elementary properties of the order $\leqq$ in an inverse semigroup (see [3, Section V.2])

$$
\left(\alpha^{-1} \beta \alpha\right)^{k}=\alpha^{-1} \beta\left(\alpha \alpha^{-1}\right) \beta \ldots\left(\alpha \alpha^{-1}\right) \beta \alpha \leqq \alpha^{-1} \beta^{k} \alpha=0
$$

hence $\left(\alpha^{-1} \beta \alpha\right)^{k}=0$.

\section{The inverse semigroup generated by $N_{1}$}

For each $\alpha$ in $H_{i, j}\left(\subset J_{n-1}\right)$ there exists a unique completion $\bar{\alpha}$ in $S_{n}$ (the symmetric group on $Z=\{1, \ldots, n\})$ defined by

$$
i \bar{\alpha}=j, \quad x \bar{\alpha}=x \alpha \quad(x \neq i) .
$$

The completion of the nilpotent $\left\|a_{1} a_{2} \ldots a_{n}\right\|$ in $J_{n-1}$ is the cycle $\left(a_{1} a_{2} \ldots a_{n}\right)$ in $S_{n}$.

From Lemma 2.5 we can see that

$$
H_{i, j} H_{k, l} \subseteq J_{n-2}
$$

if $j \neq k$. Also

$$
H_{i, j} H_{j, k} \subseteq H_{i, k}
$$

If $\alpha \in H_{i, j}$ and $\beta \in H_{j, k}$, then their product lies in $J_{n-1}$, and

$$
\overline{\alpha \beta}=\bar{\alpha} \bar{\beta} .
$$


For these reasons the question of whether an element of $J_{n-1}$ is expressible as a product of elements of $N_{1}$ is closely bound up with the expression of elements of $S_{n}$ as products of cycles of length $n$ (what we shall call $n$-cycles for brevity). There follow some results concerning $S_{n}$; these may well be known but we have been unable to find them in print.

An element $\alpha$ of $S_{n}$ partitions $Z=\{1, \ldots, n\}$ into two disjoint sets

$$
\text { shift } \alpha=\{x: x \alpha \neq x\}, \quad \text { fix } \alpha=\{x: x \alpha=x\} \text {. }
$$

When we refer to two permutations as disjoint we shall mean as usual that their shifts are disjoint.

We shall eventually prove that every even permutation $\alpha$ is expressible as a product of two cycles of length $n$. (These are not disjoint cycles, naturally.) It is convenient to prove something a little more precise: if fix $\alpha=\left\{x_{1}, \ldots, x_{p}\right\}$ we shall prove that $\alpha=\xi_{1} \xi_{2}$, where the $n$-cycle $\xi_{1}=\left(\ldots x_{1} x_{2} \ldots x_{p}\right)$ ends with the elements of fix $\alpha$ in some order and the $n$-cycle $\xi_{2}=\left(\ldots x_{p} x_{p-1} \ldots x_{1}\right)$ ends with the elements of fix $\alpha$ in the reverse order. We refer to such a product as a tidy product of two $n$-cycles.

Lemma 3.2. Every cycle of odd length $l \leqq n$ in $S_{n}$ is a tidy product of two $n$-cycles.

Proof. Let $\alpha=\left(a_{1} a_{2} \ldots a_{l}\right)$, where $l$ is odd, and write fix $\alpha=\left\{x_{1}, \ldots, x_{n-l}\right\}$. Then

$$
\alpha=\left(a_{1} a_{3} \ldots a_{l} a_{2} a_{4} \ldots a_{l-1} x_{1} \ldots x_{n-l}\right)\left(a_{l} a_{l-1} \ldots a_{1} x_{n-l} \ldots x_{1}\right)
$$

It is not posssible here to drop the restriction that $l$ be odd, for a product of two cycles of length $n$ is necessarily an even permutation, while a cycle of even length is an odd permutation. However, we do have

Lemma 3.3. If $\xi_{1}, \xi_{2}$ are disjoint cycles of even length then $\xi_{1} \xi_{2}$ is expressible as a tidy product $\eta_{1} \eta_{2}$ of two n-cycles.

Proof. Write $\xi_{1}=\left(a_{1} a_{2} \ldots a_{2 k}\right), \xi_{2}=\left(b_{1} b_{2} \ldots b_{2 l}\right)$. We may assume without loss of generality that $k \leqq l$. Let

$$
\text { fix } \xi_{1} \xi_{2}=X \backslash\left(\left\{a_{1}, \ldots, a_{2 k}\right\} \cup\left\{b_{1}, \ldots, b_{21}\right\}\right)=\left\{x_{1}, \ldots, x_{p}\right\},
$$

where $p=n-2 k-2 l \geqq 0$. Let

$$
\begin{aligned}
& \eta_{1}=\left(b_{2 l} b_{2 l-1} \ldots b_{2 k+1} a_{1} b_{1} a_{2} b_{2} \ldots a_{2 k} b_{2 k} x_{1} x_{2} \ldots x_{p}\right) \\
& \eta_{2}=\left(b_{2 k+1} b_{2 k+3} \ldots b_{2 l-1} b_{1} a_{2} b_{2} \ldots a_{2 k} b_{2 k} a_{1} b_{2 k+2} b_{2 k+4} \ldots b_{2 l} x_{p} x_{p-1} \ldots x_{1}\right)
\end{aligned}
$$

then it is not hard to verify that the tidy product $\eta_{1} \eta_{2}$ coincides with $\xi_{1} \xi_{2}$.

Lemma 3.4. Let $\sigma, \tau$ be disjoint permutations in $S_{n}$ and suppose that each of $\sigma, \tau$ is $a$ tidy product of two n-cycles. Then $\sigma \tau$ is a tidy product $\zeta_{1} \zeta_{2}$ of two n-cycles. 
Proof. Suppose that shift $\sigma=\left\{a_{1}, \ldots, a_{k}\right\}$, shift $\tau=\left\{c_{1}, \ldots, c_{l}\right\}$, fix $\sigma \tau=\left\{x_{1}, \ldots, x_{p}\right\}$. Then

$$
\text { fix } \sigma=\left\{c_{1}, \ldots, c_{l}, x_{1}, \ldots, x_{p}\right\}, \text { fix } \tau=\left\{a_{1}, \ldots, a_{k}, x_{1}, \ldots, x_{p}\right\}
$$

We may assume that we have tidy products as follows:

$$
\begin{aligned}
& \sigma=\left(a_{1} \ldots a_{k} c_{1} \ldots c_{l} x_{1} \ldots x_{p}\right)\left(b_{1} \ldots b_{k} x_{p} \ldots x_{1} c_{l} \ldots c_{1}\right) \\
& \tau=\left(c_{1} \ldots c_{l} a_{1} \ldots a_{k} x_{1} \ldots x_{p}\right)\left(d_{1} \ldots d_{l} x_{p} \ldots x_{1} a_{k} \ldots a_{1}\right)
\end{aligned}
$$

here $\left(b_{1}, \ldots, b_{k}\right),\left(d_{1}, \ldots, d_{l}\right)$ are permutations respectively of $\left(a_{1}, \ldots, a_{k}\right),\left(c_{1}, \ldots, c_{l}\right)$. Then

$$
\sigma \tau=\left(c_{1} \ldots c_{l} a_{1} \ldots a_{k} x_{1} \ldots x_{p}\right)\left(b_{1} \ldots b_{k} d_{1} \ldots d_{l} x_{p} \ldots x_{1}\right)
$$

a tidy product of two $n$-cycles. The verification of this is for the most part routine and we shall confine ourselves to noting some crucial points. If for brevity we write (3.5) as $\sigma=\xi_{1} \xi_{2}$ and (3.6) as $\tau=\eta_{1} \eta_{2}$, notice that

$$
\begin{aligned}
b_{k} & =x_{p} \xi_{2}^{-1}=x_{p} \sigma \xi_{2}^{-1} \quad\left(\text { since } x_{p} \in \text { fix } \sigma\right) \\
& =x_{p} \xi_{1}=a_{1}
\end{aligned}
$$

similarly $d_{l}=c_{1}$. Also

$$
a_{k} \sigma=c_{1} \xi_{2}=b_{1}
$$

and similarly $c_{l} \tau=d_{1}$. Then for example we may consider $a_{i}(1 \leqq i \leqq k-1)$. Using (3.5) and (3.6) we see that

$$
\begin{aligned}
a_{i} & \rightarrow a_{i+1}=b_{r} \text { (say), where } r \neq k \text { since } b_{k}=a_{1} \\
& \rightarrow b_{r+1}=a_{s} \text { (say) } \\
& \rightarrow a_{s+1} \text { (provided } s \neq k \text {; if } s=k \text { then } a_{s} \rightarrow x_{1} \text { ) } \\
& \rightarrow a_{s} .
\end{aligned}
$$

On the other hand, using (3.7) we see that

$$
a_{i} \rightarrow a_{i+1}=b_{r} \rightarrow b_{r+1}=a_{s}
$$

The other verifications are no harder than this.

As a consequence of the three lemmas proved above, we have 
Theorem 3.8. Every even permutation of $S_{n}$ is a product of at most two n-cycles.

Proof. Let $\alpha$ be an even permutation. We consider the standard decomposition of $\alpha$ into a product of disjoint cycles. If $\alpha$ is an $n$-cycle (which can happen if $n$ is odd) then no further argument is necessary; otherwise the decomposition of $\alpha$ must involve an even number of cycles of even length. The result now follows from Lemmas 3.2, 3.3 and 3.4 , and we may even conclude that the product $\alpha=\xi_{1} \xi_{2}$ is tidy.

Theorem 3.9. Let $n$ be even. Then every permutation in $S_{n}$ can be expressed as a product of at most three n-cycles.

Proof. In view of the last theorem we need only consider an odd permutation $\pi$. Then

$$
\begin{aligned}
\pi & =[(12)(134 \ldots n)]\left[(134 \ldots n)^{-1}(12) \pi\right] \\
& =(12 \ldots n) \pi^{\prime}
\end{aligned}
$$

where $\pi^{\prime}=(134 \ldots n)^{-1}(12) \pi$, being even $\times$ odd $\times$ odd, is even. The result now follows by Theorem 3.8 .

It is obvious that "three" is best possible in this result, provided $n \geqq 4$. For a product of two $n$-cycles must be an even permutation, and clearly not every odd permutation is an $n$-cycle.

We now apply these group-theoretical results to the problem of finding $\left\langle N_{1}\right\rangle$. First, we have

Lemma 3.10. Let $n$ be odd and let $\alpha \in J_{n-1}$. Then $\alpha \in\left\langle N_{1}\right\rangle$ if and only if its completion $\bar{\alpha}$ is an even permutation of $\{1, \ldots, n\}$.

Proof. Suppose first that $\bar{\alpha}$ is even, and let $\alpha \in H_{i, j}$. Then $\bar{\alpha}$ is either an $n$-cycle or a product of two $n$-cycles. In the former case $\alpha \in N_{1} \subseteq\left\langle N_{1}\right\rangle$. Otherwise $\bar{\alpha}=\xi \eta$. If we write $i \xi=p$ then we must have $p \eta=j$, since $i \bar{\alpha}=j$. Let $\gamma \in H_{i, p}, \delta \in H_{p, j}$ be such that $\bar{\gamma}=\xi, \delta=\eta$. Then $\gamma, \delta \in N_{1}$ and $\alpha=\gamma \delta$ as required. have

Conversely, suppose that $\alpha=\gamma_{1} \ldots \gamma_{k}$ for some $\gamma_{1}, \ldots, \gamma_{k}$ in $N_{1}$. Since $\alpha \in J_{n-1}$ we must

$$
\gamma_{1} \in H_{i, p_{1}}, \gamma_{2} \in H_{p_{1}, p_{2}}, \ldots, \gamma_{k} \in H_{p_{k-1}, j}
$$

for some $i, p_{1}, \ldots, p_{k-1}, j$ in $Z$. Hence $\bar{\alpha}=\bar{\gamma}_{1} \ldots \bar{\gamma}_{k}$, a product of $n$-cycles. Since $n$ is odd these $n$-cycles are even and so $\bar{\alpha}$ is even.

From the first part of this proof we have

Corollary 3.11. If $n$ is odd then

$$
\left\langle N_{1}\right\rangle \cap J_{n-1} \subseteq N_{1} \cup N_{1}^{2} \text {. }
$$


For a given $H_{i, j}$ the set $\left\{\bar{\alpha}: \alpha \in H_{i, j}\right\}$ consists of all the permutations in $S_{n}$ sending $i$ to $j$. Exactly half of the elements in each $H_{i, j}$ are in $\left\langle N_{1}\right\rangle$, and so

$$
\left|\left\langle N_{1}\right\rangle \cap J_{n-1}\right|=\frac{1}{2}\left|J_{n-1}\right|=\frac{1}{2} n^{2}(n-1) !
$$

If $n$ is even we have a different answer.

Lemma 3.12. If $n$ is even then $J_{n-1} \subset\left\langle N_{1}\right\rangle$.

Proof. Let $\alpha \in H_{i, j} \subset J_{n-1}$. Then $\bar{\alpha}$, by Theorem 3.9, is a product of one, two or three $n$-cycles. Arguing as in the first part of the proof of Lemma 3.10, we deduce that $\alpha$ is a product of one, two or three elements of $N_{1}$.

By analogy with Corollary 3.11 we have

Corollary 3.13. If $n$ is even then

$$
J_{n-1} \subset N_{1} \cup N_{1}^{2} \cup N_{1}^{3}
$$

From (3.1) it is clear that $\left\langle N_{1}\right\rangle$ contains elements of height less than $n-1$. The following lemma is helpful.

Lemma 3.14. Let $\alpha \in S P_{n}$, with $h(\alpha) \leqq n-2$. Then there exists $\xi$ in $N_{1}$ and $\beta$ in $S P_{n}$ such that $h(\beta)=h(\alpha)+1$ and $\alpha=\beta \xi$.

Proof. Write $h(\alpha)=h$ and

$$
\alpha=\left(\begin{array}{ll}
a_{1} & a_{2} \ldots a_{h} \\
b_{1} & b_{2} \ldots b_{h}
\end{array}\right)
$$

Let $x \in X \backslash\left\{a_{1}, \ldots, a_{h}\right\}$ and let

$$
X \backslash\left\{b_{1}, \ldots, b_{h}\right\}=\left\{y_{1}, \ldots, y_{n-h}\right\},
$$

where $n-h \geqq 2$ by assumption. Define

$$
\begin{gathered}
\beta=\left(\begin{array}{llll}
a_{1} & a_{2} \ldots a_{h-1} & a_{h} & x-h \\
b_{2} & b_{3} \ldots b_{h} & y_{1} & y_{n-h}
\end{array}\right), \\
\xi=\left\|y_{1} b_{h} b_{h-1} \ldots b_{1} y_{2} \ldots y_{n-h}\right\| ;
\end{gathered}
$$

then $h(\beta)=h+1, \xi \in N_{1}$, and $\alpha=\beta \xi$.

Next, we have

Lemma 3.15. $J_{n-2} \subseteq N_{1}^{2}$. 
Proof. Let $\alpha \in J_{n-2}$. Suppose in fact that

$$
\operatorname{dom} \alpha=X \backslash\{p, q\}, \quad \operatorname{im} \alpha=X \backslash\{r, s\},
$$

with $p \neq q, r \neq s$. We can still "complete" $\alpha$ to make a permutation, but there are now two possible completions $\bar{\alpha}_{1}, \bar{\alpha}_{2}$, where

$$
\begin{array}{ll}
p \bar{\alpha}_{1}=r, & q \bar{\alpha}_{1}=s, \\
p \bar{\alpha}_{2}=s, & q \bar{\alpha}_{2}=r .
\end{array}
$$

Since $\bar{\alpha}_{2}=\bar{\alpha}_{1}(r s)$, exactly one of $\bar{\alpha}_{1}, \bar{\alpha}_{2}$ is an even permutation: let $\bar{\alpha}$ be the unique even completion of $\alpha$. By Theorem 3.9 we have $n$-cycles $\zeta, \tau$ such that $\bar{\alpha}=\zeta \tau$. This applies even if $\bar{\alpha}$ is itself an $n$-cycle, for that can happen if $n$ is odd, and Lemma 3.2 makes it clear that a cycle of odd length $n$ can be expressed as a product of two $n$-cycles. Now let

$$
\gamma \in H_{p, p \zeta}, \quad \delta \in H_{q \zeta, q \bar{\alpha}}
$$

be such that $\bar{\gamma}=\zeta, \delta=\tau$. Then $\alpha=\gamma \delta \in N_{1}^{2}$ as required.

Remark 3.16. In the next section we shall require a slightly modified version of this result. In the above proof we took

$$
\gamma=\zeta|(Z \backslash\{p\}), \quad \delta=\tau|(Z \backslash\{q \zeta\}) .
$$

If instead we take

$$
\gamma^{\prime}=\zeta\left|(Z \backslash\{p, q\}), \quad \delta^{\prime}=\tau\right|(Z \backslash\{p \zeta, q \zeta\})
$$

we again obtain $\alpha=\gamma^{\prime} \delta^{\prime}$, but $\gamma^{\prime}, \delta^{\prime}$ are now in $N \cap J_{n-2}$. Thus

$$
J_{n-2} \subseteq\left(N \cap J_{n-2}\right)^{2} \text {. }
$$

From Lemmas 3.14 and 3.15 we have

Corollary 3.17. If $h(\alpha)=n-r$ then $\alpha \in N_{1}^{r}$.

Our main result is now clear:

Theorem 3.18. For $n \geqq 3$ let $S P_{n}$ be the inverse semigroup of all proper subpermutations of $\{1, \ldots, n\}$, and let $N_{1}$ be the set of all nilpotents of height $n-1$ in $S P_{n}$.

(i) If $n$ is even then

$$
\left\langle N_{1}\right\rangle=N_{1} \cup \cdots \cup N_{1}^{n}=\{0\} \cup N_{1} \cup \cdots \cup N_{1}^{n-1}=S P_{n} .
$$

(ii) If $n$ is odd then

$$
\left\langle N_{1}\right\rangle=N_{1} \cup \cdots \cup N_{1}^{n}=\{0\} \cup N_{1} \cup \cdots \cup N_{1}^{n-1}=S P_{n} \backslash W_{n-1},
$$


where $W_{n-1}$ consists of those elements of height $n-1$ whose completions in $S_{n}$ are odd permutations.

Remark 3.19. Because of Lemma 2.5 the result of Corollary 3.17 is best possible in the sense that $h(\alpha)=n-r$ implies that $\alpha \notin N_{1}^{r-1}$. Hence the result of Theorem 3.18 is also best possible. That is

$$
\left\langle N_{1}\right\rangle \neq N_{1} \cup \cdots \cup N_{1}^{n-1},\left\langle N_{1}\right\rangle \neq\{0\} \cup N_{1} \cup \cdots \cup N_{1}^{n-2} .
$$

\section{The inverse subsemigroup generated by all nilpotent elements}

From (2.4) it is clear that no product of elements (nilpotent or otherwise) in $J_{0} \cup \cdots \cup J_{n-2}$ can lie in $J_{n-1}$. Since $\left\langle N_{1}\right\rangle$ contains $J_{0} \cup \cdots \cup J_{n-2}$ we thus have $\langle N\rangle=\left\langle N_{1}\right\rangle$ and so in one sense we gain nothing by using the whole of $N$ as a set of generators rather than $N_{1}=N \cap J_{n-1}$. If we define $\Delta(\langle N\rangle)$ to be the unique $k$ such that

$$
\langle N\rangle=N \cup N^{2} \cup \cdots \cup N^{k},\langle N\rangle \neq N \cup N^{2} \cup \cdots \cup N^{k-1},
$$

then from Theorem 3.18 we deduce that

$$
\Delta(\langle N\rangle) \leqq n
$$

We shall see that this is in fact a very poor bound.

First, we have

Lemma 4.1. Let $r \in\{2, \ldots, n-1\}$. If $J_{r} \subseteq\left(N \cap J_{r}\right)^{k}$ then $J_{r-1} \subseteq\left(N \cap J_{r-1}\right)^{k}$.

Proof. Suppose that $J_{r} \subseteq\left(N \cap J_{r}\right)^{k}$, and let $\alpha \in J_{r-1}$. Let $p \in Z \backslash \operatorname{dom} \alpha, q \in Z \backslash \operatorname{im} \alpha$ and define $\alpha^{*} \in J_{r}$ by

$$
x \alpha^{*}=x \alpha \quad(x \in \operatorname{dom} \alpha), \quad p \alpha^{*}=q .
$$

By hypothesis, $\alpha^{*}=\gamma_{1}^{*} \ldots \gamma_{k}^{*}$, a product of $k$ nilpotents of height $r$. Write

$$
p \gamma_{1}^{*}=t_{2}, \quad t_{2} \gamma_{2}^{*}=t_{3}, \ldots, t_{k-1} \gamma_{k-1}^{*}=t_{k}
$$

then $t_{k} \gamma_{k}^{*}=p \gamma_{1}^{*} \ldots \gamma_{k}^{*}=p \alpha^{*}=q$. Define

$$
\gamma_{1}=\gamma_{1}^{*}\left|\left(\operatorname{dom} \gamma_{i}^{*} \backslash\{p\}\right), \quad \gamma_{i}=\gamma_{i}^{*}\right|\left(\operatorname{dom} \gamma_{i}^{*} \backslash\left\{t_{i}\right\}\right)
$$

$(i=2, \ldots, k)$. Then $\gamma_{1}, \ldots, \gamma_{k}$ are nilpotents in $J_{r-1}$ and $\alpha=\gamma_{1} \ldots \gamma_{k}$ as required.

If $n$ is even then by Corollary 3.13

$$
J_{n-1} \subseteq\left(N \cap J_{n-1}\right) \cup\left(N \cup J_{n-1}\right)^{2} \cup\left(N \cap J_{n-1}\right)^{3},
$$


and from the remark following the proof of Theorem 3.9 it is clear that 3 is best possible. If $n$ is odd then Corollary 3.11 gives

$$
\langle N\rangle \cap J_{n-1} \subseteq\left(N \cap J_{n-1}\right) \cup\left(N \cap J_{n-1}\right)^{2} \text {. }
$$

From the Remark 3.16 we know that for all $n$

$$
J_{n-2} \subseteq\left(N \cap J_{n-2}\right)^{2}
$$

Hence from Lemma 4.1 we may now deduce

Theorem 4.2. For $n \geqq 3$ let $S P_{n}$ be the inverse semigroup of all proper subpermutations of $\{1, \ldots, n\}$, and let $N$ be the set of all nilpotents in $S P_{n}$. Let $\Delta(\langle N\rangle)$ be the unique $k$ such that

$$
\langle N\rangle=N \cup N^{2} \cup \cdots \cup N^{k} \neq N \cup N^{2} \cup \cdots \cup N^{k-1} .
$$

Then $\Delta(\langle N\rangle)=2$ or 3 according as $n$ is odd or even.

\section{REFERENCES}

1. P. J. Cameron and M. Deza, on permutation geometries, J. London Math. Soc. (2) 20 (1979), 373-386.

2. A. H. Clifford and G. B. Preston, The Algebraic Theory of Semigroups, Vol. II (American Math. Soc., Providence, 1967).

3. John M. Howie, An Introduction to Semigroup Theory (Academic Press, London, 1976).

4. John M. Howie and M. Paula O. Marques-Smith, Inverse semigroups generated by nilpotent transformations, Proc. Royal Soc. Edinburgh A, 99 (1984), 153-162.

5. A. E. Liber, On symmetric generalized groups, Mat. Sbornik (N.S.) 33 (1953), 531-544 (Russian).

6. A. I. MaL'CEv, Symmetric groupoids, Mat. Sbornik (N.S.) 31 (1952), 136-151 (Russian).

7. Mario Petrich, Inverse Semigroups (Wiley, New York, 1984).

Departamento de Matemática

Faculdade de Ciencias

UNIVERSIDADE DE LISBOA

1600 Lisboa, Portugal
Mathematical Institute

UNIVERSITY OF ST ANDREWS

North HaUgh

St Andrews, Scotland 